\title{
Irrelevance between the Induction of Anti-Campylobacter Humoral Response by a Bacterin and the Lack of Protection against Homologous Challenge in Japanese Jidori Chickens
}

\author{
Masashi OKAMURA ${ }^{1) *}$, Ayumi TOMINAGA ${ }^{1)}$, Michihiro UEDA ${ }^{1)}$, Rumiko OHSHIMA ${ }^{1)}$, Masahide KOBAYASHI $^{1,2)}$, \\ Mari TSUKADA $^{1)}$, Eriko YOKOYAMA ${ }^{2)}$, Kazuaki TAKEHARA ${ }^{3)}$, Kazuhiro DEGUCHI ${ }^{2)}$, Takashi HONDA ${ }^{2)}$ and \\ Masayuki NAKAMURA ${ }^{4}$ \\ 1) Laboratory of Zoonoses, Kitasato University School of Veterinary Medicine, Towada, Aomori 034-8628, Japan \\ ${ }^{2}$ The Chemo-Sero-Therapeutic Research Institute, 1-6-1 Okubo, Kumamoto 860-8568, Japan \\ ${ }^{3)}$ Laboratory of Veterinary Hygiene, Tokyo University of Agriculture and Technology, Fuchu, Tokyo 183-8538, Japan \\ ${ }^{4)}$ Research Institute for Animal Science in Biochemistry \& Toxicology, 3-7-11 Hashimotodai, Midori-ku, Sagamihara, Kanagawa 252- \\ 0132, Japan
}

(Received 15 June 2011/Accepted 2 August 2011/Published online in J-STAGE 12 August 2011)

\begin{abstract}
On-farm vaccination of chickens against Campylobacter jejuni is considered a potentially effective countermeasure to decrease campylobacteriosis via consumption of contaminated chicken meat, but is not yet available. In this study, 2 groups of Jidori chicks were immunized subcutaneously with a formalin-killed C. jejuni with 2 different adjuvants. Other chicks served as the unvaccinated control group. Both vaccines induced high levels of anti-Campylobacter IgG but did not decrease bacterial excretion in cecal droppings and bacterial load in the liver and spleen after oral challenge with $10^{5} \mathrm{CFU}$ of the homologous strain. Further study is needed to address the observed irrelevance and to develop a novel effective vaccine against $C$. jejuni.

KEY WORDS: Campylobacter jejuni, humoral immune response, killed vaccine.
\end{abstract}

doi: 10.1292/jvms.11-0286; J. Vet. Med. Sci. 74(1): 75-78, 2012

Campylobacter jejuni, a Gram-negative, highly motile bacteria species, has recently been a major cause of infectious enteritis in humans, which is an important public health concern [1]. A major infection source of human campylobacteriosis is consumption of undercooked and/or raw chicken meat derived from Campylobacter-contaminated flocks and/or carcasses [11]. In Japan, the custom of eating raw chicken meat and organs such as the gizzard and liver is considered an important risk factor for human campylobacteriosis [4]. Recently, a number of new commercialized local brands of chickens, called "Jidori," have become available in Japan [9]. This type of chicken needs to be grown for 3-4 months (longer than broilers, which require approx. 50 days), and the meat is more palatable. Therefore, although it is not recommended, Jidori chicken meat tends to be eaten raw and might present a potentially higher risk for human campylobacteriosis than that of broilers. If so, this type of chicken should be a primary target for on-farm control of Campylobacter. On the other hand, onfarm vaccination of poultry would reduce the risk of human campylobacteriosis and have a significant impact on food safety and public health [3], but it has not yet been applied. Killed vaccines are not available for broilers due to the potential occurrence of oil cysts and vaccine residue on the

\footnotetext{
* Correspondence to: Okamura, M., Laboratory of Zoonoses, Kitasato University School of Veterinary Medicine, Towada, Aomori 034-8628, Japan.

e-mail: okamuram@vmas.kitasato-u.ac.jp

(C)2012 The Japanese Society of Veterinary Science
}

inoculation site, but they could be used for Jidori chickens, which have a longer rearing period. In the current study, we used the Japanese Jidori chicken to evaluate a prototype killed vaccine for its efficacy in decreasing the excretion and colonization of homologous challenge strains after experimental infection.

C. jejuni PD-316 (serotype of Lior 4 and Penner B, originated from a human case) was used, since serotype Lior 4 was reported to contain the largest number of strains of $C$. jejuni derived from sporadic diarrhea cases during 19982004 [7], and Penner B was predominant in broilers and layers during 2001-2006 [5] in Japan. An isolated colony grown on a modified charcoal cefoperazone deoxycholate agar (mCCDA, Oxoid, Basingstoke, UK) plate was spread on Mueller-Hinton agar (Oxoid) supplemented with 5\% defibrinated horse blood. The grown colonies were collected in phosphate buffered saline (PBS) and diluted to approximately $10^{9} \mathrm{CFU} / \mathrm{ml}$ according to an optical density at $600 \mathrm{~nm}$. The viable count was confirmed by spreading the serial 10-fold dilutions on mCCDA plates in duplicate. If not otherwise specified, the bacteria were cultured for 48 $\mathrm{hr}$ at $37.0^{\circ} \mathrm{C}$ in a microaerobic environment.

For vaccine formulation, the bacterial suspension in PBS was inactivated by mixing with the same volume of $1 \%$ formalin, kept at room temperature for $24 \mathrm{hr}$, and then supplemented with oil (bacterin, light liquid paraffin, sorbitan monooleate and polyoxyethylene sorbitan monooleate at 9:36:4:1) or aluminum hydroxide gel adjuvants (bacterin, 9 $\mathrm{mg} / \mathrm{m} l$ aluminum hydroxide gel and PBS at 9:10:31). The 
final concentration of inactivated $C$. jejuni in the vaccines was $2.7 \times 10^{8} \mathrm{CFU} / \mathrm{ml}$.

The breed of Jidori chicken used in this study is a triplecross of Shamo males and F1 females produced by crossing New Hampshire males and Barred Plymouth Rock females $(\mathrm{SH} \times[\mathrm{NH} \times \mathrm{BP}])$. One-day old chicks were divided into 3 groups of 17 chicks each and reared separately in pine shavings in an isolation building. Feed and water were provided ad libitum. Two groups of chicks were vaccinated subcutaneously in the leg $(0.5 \mathrm{ml} /$ chick): one received an oil adjuvant vaccine at 37 days old, and the other received an aluminum gel adjuvant vaccine at 37 and 58 days old. The remaining 17 birds served as the unvaccinated control group. The chicks were confirmed to be Campylobacterfree by examining fresh cecal droppings and challenged orally with $10^{5} \mathrm{CFU}$ of $C$. jejuni PD316 at 72 days old. Bacterial excretion in cecal droppings $(77,85,91$ and 111 days old) was then monitored. When 5 and 12 birds were necropsied at 98 and 128 days old, respectively, the viable counts of Campylobacter were obtained for the liver, spleen and cecal contents. This animal experiment was approved by the Animal Research Committee, Kitasato University School of Veterinary Medicine, Japan. For bacterial examination, each sample taken was weighed, mixed with nine volumes of Campylobacter enrichment medium (CEM) [8] and stomached. The serial 10 -fold dilutions were spread on mCCDA plates in duplicate and cultured. The numbers of colonies were enumerated and transformed to log values (detection limit: $\log _{10} 400 \mathrm{CFU} / \mathrm{g}$ ). In addition, CEM-mixed samples were incubated for $24 \mathrm{hr}$ for enrichment. The bacterial counts of positive samples after enrichment culture were assumed to be $1 / 2 \log _{10} 400 / \mathrm{g}$. The enumerated CFU data were compared between each vaccinated group and unvaccinated hens by the unpaired Student's $t$-test. As a result, the level of bacterial excretion in the postchallenge cecal droppings was in the range of $10^{6}-10^{8} \mathrm{CFU} / \mathrm{g}$, and there was no difference $(P>0.05)$ between the vaccinated and unvaccinated birds (data not shown). No $C$. jejuni was isolated from the livers or spleens sampled at 26 and 56 days postchallenge. Although C. jejuni was isolated from the cecal content, no statistical difference $(P>0.05)$ was observed between the vaccinated and unvaccinated birds (Fig. 1).

Serum IgG levels $(37,44,58,72,86,98,114$ and 128 days old) against Campylobacter were also measured by enzyme-linked immunosorbent assay (ELISA) using Campylobacter outer membrane antigen solution [17] as a coating antigen $(6 \mathrm{ng} / \mathrm{ml})$. Serum samples were diluted 100fold and applied (100 $\mu / /$ well). After reaction with a rabbit anti-chicken IgG conjugated with peroxidase $(1: 10,000$, Sigma; $100 \mu l /$ well), the reaction was colored with peroxidase substrate (100 $\mu l /$ well) and stopped using $2 \mathrm{~N} \mathrm{H}_{2} \mathrm{SO}_{4}$ $(50 \mu l / w e l l)$. The OD values were measured at $450 \mathrm{~nm}$. The data were analyzed by the unpaired Student's $t$-test as described above. Both vaccinated groups showed increased IgG levels against $C$. jejuni outer membrane components after vaccination, which were higher than those of control

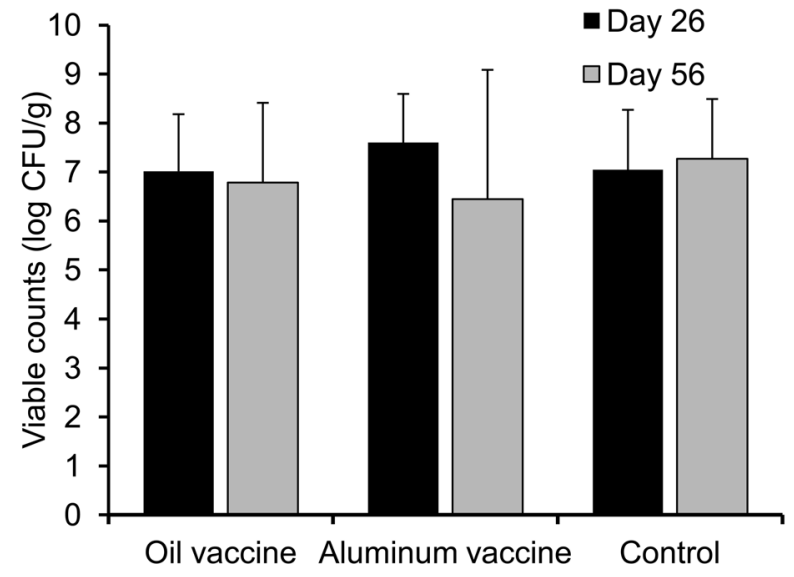

Fig. 1. Recovery of $C$. jejuni from cecal contents at necropsies. Five and 12 birds from each group were necropsied at 26 and 56 days post challenge, respectively. The liver, spleen and cecal contents were aseptically sampled, and bacterial counts were enumerated. The bacteria were recovered from cecal content only. There were no significant differences between the vaccine groups and control group at any time point.

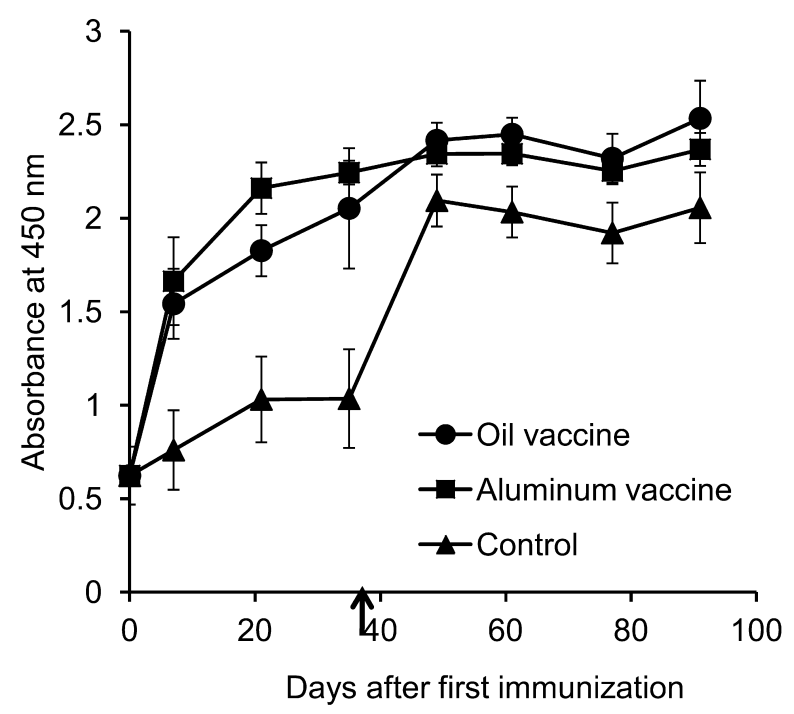

Fig. 2. C. jejuni-specific IgG levels after immunization. The IgG antibodies to the Campylobacter outer membrane components in sera collected at $0,7,21,35,49,61,77$ and 91 days post first vaccination were measured by enzyme-linked immunosorbent assay. Arrow indicates the date of challenge. There were significant differences between the vaccine groups and control group at all time points $(P<0.01)$.

birds throughout the experimental period $(P<0.01$; Fig. 2). In an earlier study by Rice et al. [14], immunization of broilers with formalin-inactivated $C$. jejuni containing $10^{9} \mathrm{CFU}$ resulted in a limited reduction of $C$. jejuni from $1.4 \times 10^{9}$ to $1.0 \times 10^{8} \mathrm{CFU} / \mathrm{g}$ in the cecum after a homologous challenge. A strong correlation between increased levels of antibodies against $C$. jejuni and reduced shedding of the bacteria in 
chickens $[2,12,14]$ and a potential contribution of maternal antibodies to delayed colonization after 2-3 weeks of age observed under field conditions [16] encouraged the development of an effective vaccine against $C$. jejuni in chickens. In this study, however, the prototype vaccines induced a high level of antibody against $C$. jejuni but did not provide protection in Jidori chickens.

To determine which antigens of $C$. jejuni were recognized by vaccine-induced serum antibodies, western blotting analysis was performed. The Campylobacter outer membrane antigens used for ELISA were applied to sodium dodecyl sulfate-polyacrylamide gel electrophoresis (SDS-PAGE) [10]. The separated proteins were stained with Coomassie Brilliant Blue or transferred onto a nitrocellulose membrane (Amersham Bioscience, Little Chalfont, Bucks HP7 9NA, England), which was reacted with pooled sera (1:100) taken at 35 and 49 days post vaccination (just before and 14 days post challenge, respectively) after blocking. The bound antibody was detected with peroxidase-conjugated rabbit anti-chicken-IgG antibody $(1: 1,000$, ICN/Cappel, Aurora, $\mathrm{OH}$, U.S.A.) and the peroxidase substrate (Roche Applied Science, Mannheim, Germany). The resultant banding patterns obtained from both vaccinated groups were almost identical before and after the challenge, showing five major bands estimated at 100,65, 59, 45 and $29 \mathrm{kDa}$ and several minor bands of 16-26 kDa (data not shown). Of these, four major bands of approximately 65, 59, 45 and $29 \mathrm{kDa}$ were also observed in the post-challenge unvaccinated control sera in our study, which were estimated to contain FlaA/B, according to the recent study by Shoaf-Sweeney et al. [18]. These antigens were considered immunogenic enough to induce antibodies but did not lead to protection, probably because of the genetic diversity [6] and glycosylated feature of the flagellar filament [19]. Sera from both groups of vaccinated birds covered the range of outer membrane components recognized by unvaccinated challenged birds (data not shown). Thus, the role of the humoral immune responses in Campylobacter infection in chickens is still controversial. In our study, the potential effect of maternal antibodies [16] should be excluded, since the level of anti-Campylobacter antibodies in chickens at 37 days of age was much lower than that measured before vaccination. Future study should focus on the intestinal secreted $\operatorname{IgA}$, which was suggested to be important for protection against Campylobacter by Widders et al. [20].

Such limited success of immunization with killed vaccines, including ours, is thought to be due to the adaptive changes of phenotypes of $C$. jejuni in vivo to better colonize and survive in the chicken intestine by its genetic instability [15]. To confirm that the isolates from tissue and cecal samples were the same as the challenge strain in our study, isolates were randomly selected and analyzed by pulsed-field gel electrophoresis (PFGE) according to the standardized PulseNet protocol [13]. Samples were digested using SmaI. However, no changes were observed in PFGE patterns between the challenge strain and those recovered from cecal droppings in our study (data not shown); therefore, this pos- sibility was excluded. Another possibility could be a potential difference in the expression of surface structures or in the secretion profile of antigens by the vaccine strain between its in vitro growth in a culture medium before inactivation and its in vivo colonization of the intestine after challenge. Examining this second possibility might be helpful in addressing, in future studies, why bacterin-induced antibodies against $C$. jejuni do not protect chickens challenged with a homologous strain.

In conclusion, the prototype killed vaccines failed to protect chickens from colonization by $C$. jejuni in spite of high induced antibody levels. However, our attempt should facilitate the future effort to address the observed irrelevance and to develop a novel effective vaccine against $C$. jejuni. Further study should also be promoted toward the development of live vaccines, which can be used for broilers, to induce a local IgA response in the host intestine and to examine use of other effective immunization routes, such as spray, eye drops and in ovo vaccination.

\section{REFERENCES}

1. Allos, B. M. 2001. Campylobacter jejuni Infections: update on emerging issues and trends. Clin. Infect. Dis 32: 1201-1206.

2. Cawthraw, S., Ayling, R., Nuijten, P., Wassenaar, T. and Newell, D. G. 1994. Isotype, specificity, and kinetics of systemic and mucosal antibodies to Campylobacter jejuni antigens, including flagellin, during experimental oral infections of chickens. Avian Dis. 38: 341-349.

3. de Zoete, M. R., van Putten, J. P. and Wagenaar, J. A. 2007. Vaccination of chickens against Campylobacter. Vaccine 25 : $5548-5557$.

4. Food-Safety-Commission. 2009. Evaluation Report on Microorganisms and Viruses: Campylobacter jejuni and Campylobacter coli in Poultry. Food Safety Commission, Tokyo (in Japanese).

5. Harada, K., Ozawa, M., Ishihara, K., Koike, R., Asai, T. and Ishikawa, H. 2009. Prevalence of antimicrobial resistance among serotypes of Campylobacter jejuni isolates from cattle and poultry in Japan. Microbiol. Immunol. 53: 107-111.

6. Harrington, C. S., Thomson-Carter, F. M. and Carter, P. E. 1997. Evidence for recombination in the flagellin locus of Campylobacter jejuni: implications for the flagellin gene typing scheme. J. Clin. Microbiol. 35: 2386-2392.

7. Infectious Disease Surveillance Center. 2006. Campylobacter enteritis in Japan, 1999-2005. Infect. Agents Survei. Rep. (IASR) 27: 167-168.

8. Itoh, T. and Sakai, S. 1984. Distribution and pathogenicity of Campylobacter in poultry. J. Jpn. Soc. Poult. Dis. 20: 2-12.

9. Japan-Chicken-Association. 2003. The Guidebook of Special Meat-Type Chickens. Zenkoku-Shokuchou-Shinbun Co., Ltd., Tokyo (in Japanese).

10. Laemmli, U. K. 1970. Cleavage of structural proteins during the assembly of the head of bacteriophage T4. Nature 227: 680-685.

11. Moore, J. E., Corcoran, D., Dooley, J. S., Fanning, S., Lucey, B., Matsuda, M., McDowell, D. A., Megraud, F., Millar, B. C., O’Mahony, R., O'Riordan, L., O'Rourke, M., Rao, J. R., Rooney, P. J., Sails, A. and Whyte, P. 2005. Campylobacter. Vet. Res. 36: 351-382.

12. Myszewski, M. A. and Stern, N. J. 1990. Influence of Campy- 
lobacter jejuni cecal colonization on immunoglobulin response in chickens. Avian Dis. 34: 588-594.

13. Ribot, E. M., Fitzgerald, C., Kubota, K., Swaminathan, B. and Barrett, T. J. 2001. Rapid pulsed-field gel electrophoresis protocol for subtyping of Campylobacter jejuni. J. Clin. Microbiol. 39: 1889-1894.

14. Rice, B. E., Rollins, D. M., Mallinson, E. T., Carr, L. and Joseph, S. W. 1997. Campylobacter jejuni in broiler chickens: colonization and humoral immunity following oral vaccination and experimental infection. Vaccine 15: 1922-1932.

15. Ridley, A. M., Toszeghy, M. J., Cawthraw, S. A., Wassenaar, T. M. and Newell, D. G. 2008. Genetic instability is associated with changes in the colonization potential of Campylobacter jejuni in the avian intestine. J. Appl. Microbiol. 105: 95-104.

16. Sahin, O., Luo, N., Huang, S. and Zhang, Q. 2003. Effect of Campylobacter-specific maternal antibodies on Campylobacter jejuni colonization in young chickens. Appl. Environ.
Microbiol. 69: 5372-5379.

17. Sahin, O., Zhang, Q., Meitzler, J. C., Harr, B. S., Morishita, T. Y. and Mohan, R. 2001. Prevalence, antigenic specificity, and bactericidal activity of poultry anti-Campylobacter maternal antibodies. Appl. Environ. Microbiol. 67: 3951-3957.

18. Shoaf-Sweeney, K. D., Larson, C. L., Tang, X. and Konkel, M. E. 2008. Identification of Campylobacter jejuni proteins recognized by maternal antibodies of chickens. Appl. Environ. Microbiol. 74: 6867-6875.

19. Thibault, P., Logan, S. M., Kelly, J. F., Brisson, J. R., Ewing, C. P., Trust, T. J. and Guerry, P. 2001. Identification of the carbohydrate moieties and glycosylation motifs in Campylobacter jejuni flagellin. J. Biol. Chem. 276: 34862-34870.

20. Widders, P. R., Perry, R., Muir, W. I., Husband, A. J. and Long, K. A. 1996. Immunisation of chickens to reduce intestinal colonisation with Campylobacter jejuni. Br. Poult. Sci. 37: 765-778. 